\title{
Early endocarditis following percutaneous mitral repair in a dialysis patient
}

\author{
Rambhujun VP ${ }^{*}$, Kennedy-Snodgrass $\mathrm{C}^{1}$ and Kerwin $\mathrm{T}^{2}$ \\ ${ }^{1}$ NYU Winthrop Internal Medicine department, Mineola, NY 11501, USA \\ ${ }^{2}$ NYU Winthrop Cardiology department, Mineola, NY 11501, USA
}

\begin{abstract}
Transcutaneous mitral valve repair is an interventional procedure increasingly used as a method of treatment for patients with symptomatic mitral regurgitation deemed to be poor surgical candidates. The procedure involves clipping the mitral valve leaflets at the point of maximal regurgitation. This procedure has been shown to be well tolerated with an acceptable risk profile. Infective endocarditis is a known but unusual complication of this procedure. We describe a case of infective endocarditis following implantation of a MitraClip (Abbott Laboratories) in a hemodialysis patient.
\end{abstract}

\section{Introduction}

Mitral regurgitation (MR) or incompetence is a condition in which there is back flow of blood into the left atrium during systole as a result of incomplete apposition of the mitral valve leaflets. The disease affects roughly $2-3 \%$ of people in industrialized countries and in 2009 it was estimated that the prevalence was about 2 million people in the US $[1,2]$.

The management of mitral regurgitation is medical initially and as the disease progresses further, the options are either surgical or transcatheter repair or mitral valve replacement depending on the severity and patient risk factors [2,3]. One of the novel interventions for treatment of severe mitral regurgitation and heart failure for patients who are very high risk surgical candidates is transcatheter mitral valve repair (TMVR) with the MitraClip [4].

The technique involves clipping the mitral valve leaflets at the point of maximal flow to create smaller orifices, thereby reducing the regurgitant flow [5]. This technique improves congestive heart failure symptoms of severe MR patients with lower periprocedural mortality $[6,7]$.

Overall the MitraClip procedure has a relatively low immediate and short-term complication rate [8]. Since the introduction of this device, very few cases of infective endocarditis have been reported [9-13]. We describe a patient who had a MitraClip implanted who one month later developed mitral valve endocarditis.

\section{Case report}

Our patient was a 76-year-old female who presented to the hospital with progressive fatigue, fever, and dislodged dialysis catheter. She had a past medical history of severe MR status post MitraClip placement one month prior to presentation, coronary artery disease status post three vessel coronary artery bypass, atrial fibrillation on oral anticoagulation with warfarin, end stage renal disease (ESRD) requiring hemodialysis, hypertension and non-insulin dependent diabetes mellitus. On presentation the patient was hypotensive, with leukocytosis WBC count 21.7 and an elevated lactate of 3.0. Given her history of extended spectrum beta-lactamase producing E. coli urinary tract infections and the indwelling catheter; she was empirically treated with vancomycin and meropenem. Blood cultures were positive for methicillinsusceptible Staphylococcus aureus (MSSA) sensitive to vancomycin and cefazolin; after which antibiotic therapy was de-escalated to cefazolin. However, blood cultures were persistently positive for MSSA, at which point a work up for the persistent bacteremia was initiated.

A transesophageal echocardiogram (TEE) demonstrated a prosthetic mitral clip which overall appeared thickened with two mobile $5 \mathrm{~mm}$ masses associated with the clip at the leaflet tips, suggestive of vegetative structures with moderate to severe $M R$.

Given the patient's overall poor condition, surgical therapy was not pursued, and the patient was treated with prolonged antibiotic therapy with vancomycin. The patient was readmitted one week later for infectious versus ischemic colitis. She presented again one month later for hypoglycemia. Her hospital course was complicated by disseminated intravascular coagulation (DIC) secondary to underlying infection and hypercapnic respiratory failure. Repeat blood cultures were negative. The patient continued to receive broad-spectrum antibiotics, however, her clinical condition continued to deteriorate, and she ultimately succumbed to her illness (Figures 1-3).

\section{Discussion}

For a select group of patients with severe MR who are at extreme risk for surgery due to advanced age, multiple comorbidities or severely reduced ejection fraction, TMVR with the MitraClip is an emerging alternative option [14]. The EVEREST II trial compared the efficacy of surgical mitral valve repair to TMVR for degenerative MR and the latter was found to be less effective at reducing the severity of MR

Correspondence to: Vikashisngh Rambhujun, MBBS, Internal Medicine resident, NYU Winthrop Internal Medicine department, Mineola, NY 11501, USA, E-mail: Vikash_rambhujun@hotmail.com

Key words: infective endocarditis, MitraClip

Received: March 15, 2018; Accepted: March 29, 2018; Published: April 03, 2018 
and more commonly required surgery to treat residual MR $[15,16]$. However, TMVR was associated with a significantly superior safety profile when compared to surgical valve repair $[15,16]$. Following these

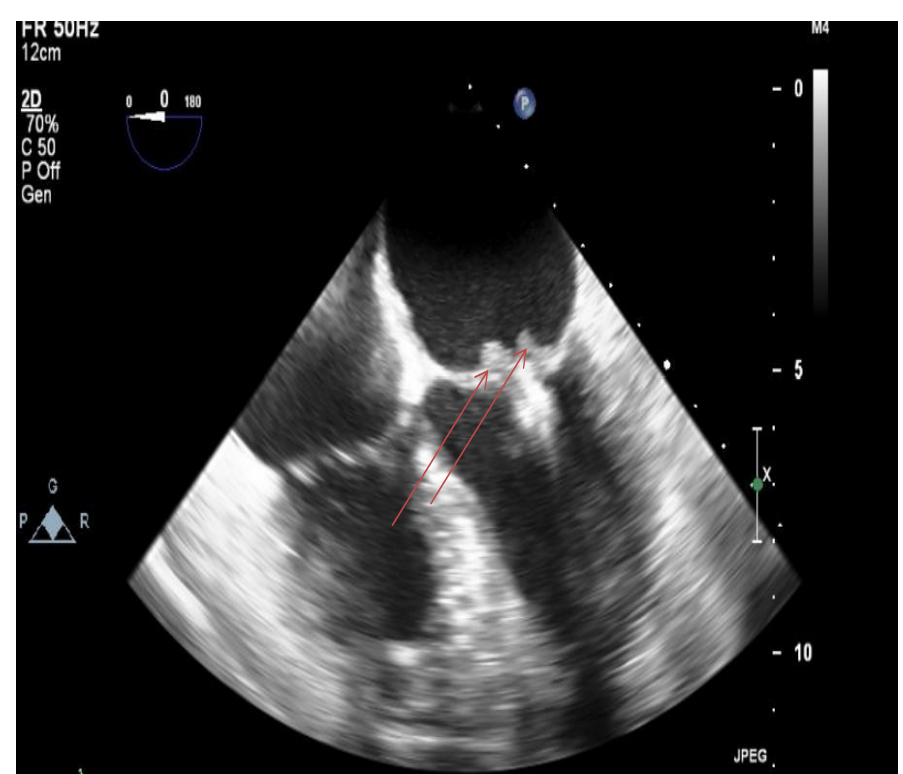

Figure 1. Transesophageal echocardiogram image demonstrating two mobile masses (highlighted by the arrows), likely vegetations, at leaflet tips one month after MitraClip placement $(7 / 19 / 17)$

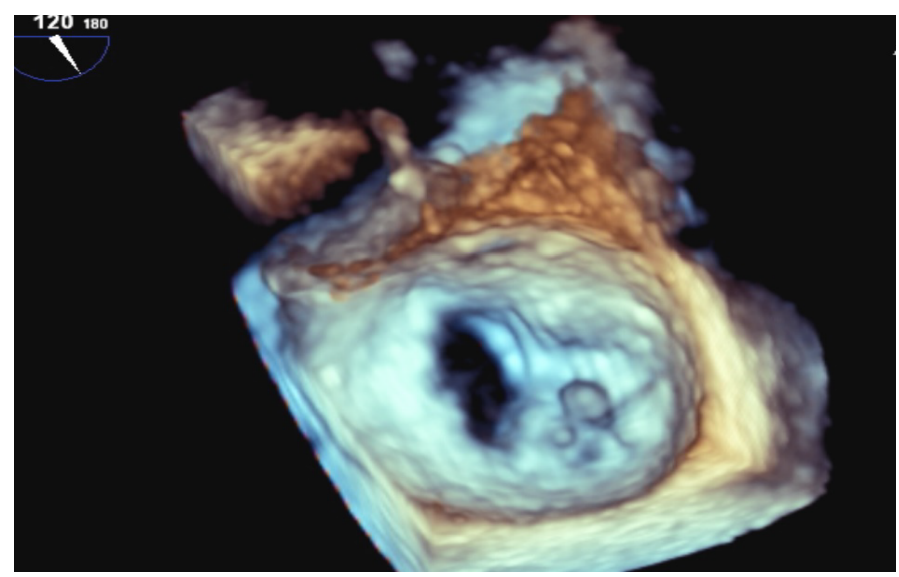

Figure 2. Shows the 3D reconstruction of the Mitral valve orifice seen from the atrial area The red arrows point to the vegetations on the Mitraclip

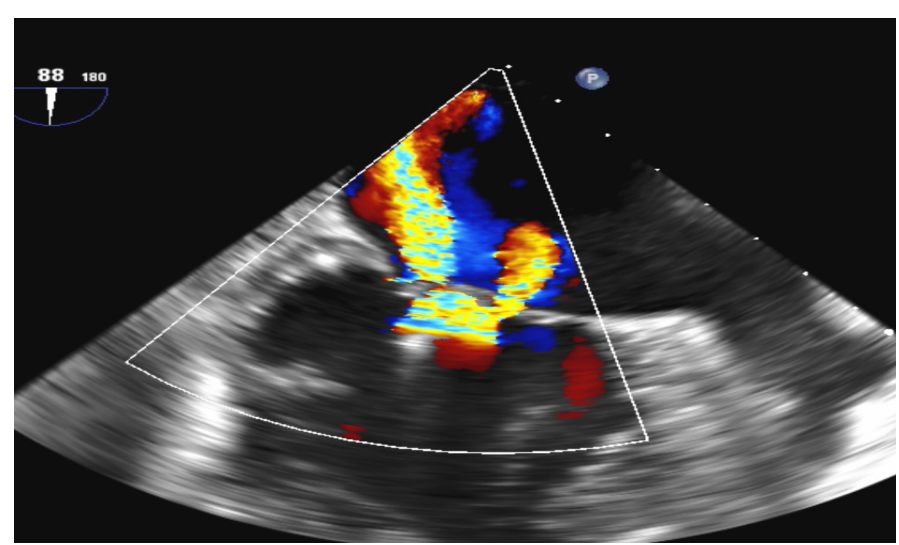

Figure 3. Shows a color Doppler of a cross sectional section of the Mitral valve with the Mitraclip in place. The picture shows severe MR on the color doppler results, the MitraClip was approved by the FDA in November of 2013 for treatment of degenerative mitral valve regurgitation in patients who are not surgical candidates or who are at very high risk [17].

Major complications reported from the EVEREST II trial and a four-year MitraClip follow up study include major bleeding, stroke or transient ischemic attack, chordal rupture, pericardial tamponade and myocardial infarction; endocarditis, however, was not one of the reported complications in the landmark EVEREST II trial $[18,19]$. There have been only a few reported cases of patients with infective endocarditis in the region of the MitraClip $[9,10]$. The exact incidence of endocarditis in patients who underwent TMVR remains to be quantified because of its novelty and the rarity of infective endocarditis following this procedure $[9,10]$.

Our patient had newly diagnosed end stage renal disease for which she had outpatient dialysis through a chronic indwelling venous catheter. Like most reported cases of MitraClips complicated by infective endocarditis, our patient presented with fever and nonspecific symptoms like progressive weakness; she was, eventually, found to have Staphylococcus aureus bacteremia. A transesophageal echocardiogram showed vegetations in the region of the MitraClip which confirmed the diagnosis.

The incidence of endocarditis in the general population is 11 to 15 cases per 100,000 people [20]; prosthetic valve endocarditis occurs in $1-6 \%$ of all patients, the risk is highest in the first 3 months and then gradually tapers down to a rate of $0.4 \%$ at 12 months [4]. In patients with End Stage Renal Disease (ESRD) requiring hemodialysis, the incidence of endocarditis of bioprosthetic or mechanical valves was $7.8 \%$ and $5.4 \%$ respectively, which is ten to fifteen times higher than the incidence of endocarditis in patients with chronic kidney disease (CKD), which was about $0.41 \%$ [21,22]. Endocarditis can be a major but rare complication of cardiac device implantation; for example, in patients with pacemakers and defibrillators, the rate of endocarditis is $0.38 \%[23]$.

\section{Conclusion}

Our patient had ESRD on hemodialysis with an indwelling catheter placed after receiving a MitraClip. The exact risk of endocarditis in MitraClip patients with ESRD with or without chronic indwelling catheters remains to be determined. This is the first reported case of a patient with a chronic indwelling catheter who developed endocarditis on the MitraClip. As this procedure becomes more frequently used for treatment of severe MR in select patients, the incidence of endocarditis, particularly in high risk subsets, such as dialysis patients, bears monitoring [24-29].

\section{References}

1. Madesis A, Tsakiridis K, Zarogoulidis $\mathrm{P}$, Katsikogiannis N, Machairiotis N, et al. (2014) Review of mitral valve insufficiency: repair or replacement. J Thorac Dis 6: S39-S51. [Crossref]

2. Enriquez-Sarano M, Akins CW, Vahanian A (2009) Mitral regurgitation. Lancet 373: 1382-1394. [Crossref]

3. Mauri L, Foster E, Glower DD, Apruzzese P, Massaro JM, et al. (2013) 4-year results of a randomized controlled trial of percutaneous repair versus surgery for mitral regurgitation. J Am Coll Cardiol 62: 317-328. [Crossref]

4. Joint Task Force on the Management of Valvular Heart Disease of the European Society of Cardiology (ESC), European Association for Cardio-Thoracic Surgery (EACTS), Vahanian A, Alfieri O, Andreotti F, et al. (2012) Guidelines on the management of valvular heart disease (version 2012). Eur Heart J 33: 2451-2496. [Crossref]

5. Baptist Health (2017) MitraClip | Heart \& Vascular Care. 
6. D'ascenzo F, Moretti C, Marra WG, Montefusco A, Omede P, et al. (2015) MetaAnalysis of the Usefulness of Mitraclip in Patients with Functional Mitral Regurgitation. Am J Cardiol 115: 325-331. [Crossref]

7. Magruder JT, Crawford TC, Grimm JC, Fredi JL, Shah AS (2016) Managing mitral regurgitation: focus on the MitraClip device. Med Devices (Auckl) 9: 53-60. [Crossref]

8. Deuschl F, Schofer N, Lubos E, Schirmer J, Conradi L, et al. (2015) MitraClip-data analysis of contemporary literature. $J$ Thorac Dis 7: 1509-1517. [Crossref]

9. Frerker C, Kuck KH, Schmidt T, Kreidel F, Bader R, et al. (2015) Severe infective endocarditis after mitraclip implantation treated by cardiac surgery. EuroIntervention 11: 351-354. [Crossref]

10. Vazir A, Franzen O, Moat NE, Price S (2013) Infective Endocarditis Associated with the MitraClip. In: Feldman T., Franzen O., Low R., Rogers J., Yeo K. (eds) Atlas of Percutaneous Edge-to-Edge Mitral Valve Repair. Springer, London.

11. Maznikoski D, Denk K, Karliova I, Vahl CF (2013) Mitral valve repair: Infective endocarditis after MitralClip. Thorac Cardiovasc Surg 61-SC132.

12. Boeder NF, Dorr O, Rixe J, Weipert K, Bauer T, et al. (2017) Endocarditis after interventional repair of the mitral valve: Review of a dilemma. Cardiovasc Revasc Med 18: 141-144. [Crossref]

13. Saito S, Baraki H, Fleischer B, Kutschka I (2015) Mitral valve replacement after failed MitraClip therapy: report of two cases. J Artif Organs 18: 177-180. [Crossref]

14. Deuschl F, Schofer N, Lubos E, Blankenberg S, Schäfer U (2016) Critical evaluation of the MitraClip system in the management of mitral regurgitation. Vasc Health Risk Manag 12: 1-8. [Crossref]

15. Feldman T, Foster E, Glower DD, Kar S, Rinaldi MJ, et al. (2011) Percutaneous repair or surgery for mitral regurgitation. $N$ Engl J Med 364: 1395-1406. [Crossref]

16. Mauri L, Foster E, Glower DD, Apruzzese P, Massaro JM, et al. (2013) 4-Year results of a randomized controlled trial of percutaneous repair versus surgery for mitral regurgitation. J Am Coll Cardiol 62: 317-328. [Crossref]

17. Ramlawi B, Gammie JS (2016) Mitral Valve Surgery: Current Minimally Invasive and Transcatheter Options. Methodist Debakey Cardiovasc J 12: 20-26. [Crossref]

18. Vakil K, Roukoz H, Sarraf M, Krishnan B, Reisman M, et al. (2014) Safety and efficacy of the MitraClip system for severe mitral regurgitation: a systemic review. Catheter Cardiovasc Interv 84: 129-136. [Crossref]
19. Eggebrecht H, Schelle S, Puls M, Plicht B, von Bardeleben RS, et al. (2015) Risk and outcomes of complications during and after MitraClip implantation: Experience in 828 patients from the German TRAnscatheter mitral valve interventions (TRAMI) registry. Catheter Cardiovasc Interv 86: 728-735. [Crossref]

20. Pant S, Patel NJ, Deshmukh A, Golwala H, Patel N, et al. (2015) Trends in infective endocarditis incidence, microbiology, and valve replacement in the United States from 2000 to 2011. J Am Coll Cardiol 65: 2070-2076. [Crossref]

21. Phan K, Zhao DF, Zhou JJ, Karagaratnam A, Phan S, et al. (2016) Bioprosthetic versus mechanical prostheses for valve replacement in end stage renal disease patients: a systemic review and meta-analysis. J Thorac Dis 8: 769-777. [Crossref]

22. Chaudry MS, Carlson N, Gislason GH, Kamper AL, Rix M, et al. (2017) Risk of infective endocarditis in patients with end stage renal disease. Clin J Am Soc Nephrol 12: 1814-1822. [Crossref]

23. Osmonov D, Ozcan KS, Erdinler I, Altay S, Yildirim E, et al. (2013) Cardiac devicerelated endocarditis: 31-Years' experience. $J$ Cardiol 61: 175-180.

24. Nishimura RA, Otto CM, Bonow RO, Carabello BA, Erwin JP III, et al. (2014) 2014 AHA/ACC guideline for the management of patients with valvular heart disease: executive summary: a report of the American College of Cardiology/American Heart Association Task Force on Practice Guidelines. Circulation 129: 54-57. [Crossref]

25. DeSimone DC, Chahal AA, DeSimone CV, Asirvatham SJ, Friedman PA, et al. (2017) International survey of knowledge, attitudes, and practices of cardiologists regarding prevention and management of cardiac implantable electronic device infections. Pacing Clin Electrophysiol 40: 1260-1268. [Crossref]

26. Podoleanu C, Deharo JC (2014) Management of Cardiac Implantable Electronic Device Infection. Arrhythm Electrophysiol Rev 3: 184-189. [Crossref]

27. Kale SB, Raghavan J (2013) Tricuspid valve endocarditis following central venous cannulation: The increasing problem of catheter related infection. Indian J Anaesth 57: 390-393. [Crossref]

28. Jilaihawi H, Hussaini A, Kar S (2011) MitraClip: a novel percutaneous approach to mitral valve repair. J Zhejiang Univ Sci B 12: 633-637. [Crossref]

29. Duval X, Delahaye F, Alla F, Tattevin P, Obadia JF, et al. (2012) Temporal Trends in Infective Endocarditis in the Context of Prophylaxis Guideline Modifications: Three Successive Population Based Surveys. J Am Coll Cardiol 59: 1968-1976. [Crossref]

Copyright: (C2018 Rambhujun VP. This is an open-access article distributed under the terms of the Creative Commons Attribution License, which permits unrestricted use, distribution, and reproduction in any medium, provided the original author and source are credited. 